\title{
Brief Discussion on The Neo-Chinese Style Living Room Soft Assembly Decoration Design
}

\author{
Xiaohui Xin \\ Department of Art and Design, Weihai Vocational College, Weihai Shandong, 264200, China
}

Keywords: The Neo-Chinese Style, Living room, Soft assembly decoration.

\begin{abstract}
After China's home design getting through a simple imitation stage, new generation of designers gradually embedded Chinese traditional culture into the modern home design style, through combination of tradition and modern to form a unique Chinese style home design concept. Neo-Chinese style home design and soft assembly design expressed Chinese peoples' chase for restrained and elegant eastern aesthetic realm, this is the most perfect expression of modern Chinese people of our five thousand years civilization to the whole world, it is the most national characteristics beautiful scenery among various styles. This paper detailed explained the design orientation, design philosophy and design path of the Neo-Chinese style.
\end{abstract}

\section{Introduction}

The main difference between the Neo-Chinese style and traditional home design is that the home design of the Neo-Chinese style integrates more modern design elements, it is a new decoration style complies with living concept and aesthetical standard. "the Neo-Chinese style" expressed Chinese traditional decoration elements, and through designers' understanding on traditional cultures to combine modern and traditional elements, combine modern technology, fully absorb excellent traditional home design culture, integrate with modern design and fashion living concept, through modern aesthetic to build living space with traditional flavor.

With the continue thorough and development of China's living design, soft assembly decoration has more and more important function for brand development, mutual collocation of different products to match unique soft assembly to create higher quality living space, even meet similar product, it also has unique style and features, this feature has huge influence on brand development.

\section{Decoration design for living room}

It is helpful improving image: excellent and suitable soft assembly decoration design not just can express design and sales information of stores to customers, but also can transfer enterprises' unique culture brand, to leave a deeper image on customers.

Enrich space: in furniture stores or exhibition room, soft assembly decoration can broaden sense of space, this can make space function become more reasonable and with sense of depth to make atmosphere for home design.

Strengthen style: because soft assembly itself has own shape, picture, color and texture, therefore, this kind of furniture design will strengthen its environmental style.

Promote sales: the purpose of living room furniture design is sale, in this kind of stores or exhibition centers for living room furniture, soft assembly decoration can fully express product beauty and quality, make quiet products become more attractive, in addition with designers' design and planning, living room's level can be improved, which makes it with more commercial value and use value. 


\section{Design orientation of the Neo-Chinese style living room soft assembly decoration}

The Neo-Chinese style living room soft assembly decoration chases for integrality, its soft outfit design language and hardware design language integrate to reach unity. The texture, shape, color, assembly detail of hardware in the living room should be coordinated, they are not exist independently, but need ingenious integration, on the other hand, they also need to be coordinated with furniture, clothes, lights, decoration and assembly on the wall, indoor plants. Make whole space in consistency and integration, make the whole living room has a strong visual perception, thus to create harmonious Neo-Chinese design style. In order to obtain this effect, one of the simplest methods is to use an article with same Chinese elements to realize integration. For example, in one of the model houses design of the very famous interior designer-Liang Zhitian, he put the window partition with Chinese flavor and furniture full of modern sense and colorful oil painting in one space, look at these elements separately, they are widely different, but they are quite harmonious when put together.

Because everyone has different interests, job, culture background, all these influence factors have different features, the Neo-Chinese style soft assembly decorations are also widely different. Therefore, designers have to communicate with proprietors effectively, to have a deep understanding on their aethestic standard and value orientation then to make design orientation. Nowadays, modern soft decoration products are rich and colorful, especially in Guangzhou, Suzhou, Wenzhou and other cities, soft decoration kinds are rich, same on online stores like Taobao and Jingdong for designers and proprietors, there are a large number of purchasing channels, which provided a huge market resources for the Neo-Chinese style to meet demands of proprietors with different culture background, job and interests.

The color orientation of Neo-Chinese style is not just limited in traditional red, black and wooden. Modern Neo-Chinese style has wide color range, both strong and light colors, red, green are strong colors, wash painting belongs to light color, there are also many middle colors among them, which has a good coordination function. Color match cannot be moderation in traditional Chinese styles, we should choose a color to play the main color in the living room. Relatively speaking, living room is a big environment, choice if any color should be serious to avoid separation, there should be colors and style matching with it. Until now, color of the Neo-Chinese style living room design mainly develops into two directions: fresh and elegant, with elegant colors in Chinese painting, the main color of this color system should be natural color and colourless, which can obviously express residents' reservation characteristics; bright, with sharp colors with Chinese flavor, mainly red, yellow, blue and green, which can reflect residents' pleasant and outgoing personality.

\section{Design method of the Neo-Chinese style living room decoration}

\section{Design of decoration}

In the Neo-Chinese living room environment design, though decoration is ornament item, it can foil atmosphere. For the arrangement of living room decorations, the arrangement of Chinese decorations must show features of significant and the lesser, use color, size, texture and light of decoration to highlight the main one, this kind of decoration should be visual center, others just for assistance. This cannot lead to disorder special effect, but can make the whole space be with more sense of space. The Neo-Chinese decoration design should pay more attention to the unique style orientation of living room style, generally speaking, there are many kinds of plane decorations, such as calligraphy, paper-cut, photos and painting; three-dimensional decorations include Chinese knot, tri-coloured glazed pottery of the Tang Dynasty, lacquerware, pottery, blue and white porcelain, mask, lantern, Chinese paper-cut for window decoration, glasswork, crystal craft, plant knit and others. And old things with historical precipitation significance, such as old radio, phonograph, barn lantern and so on. Common living room decorations include green plant, such as orchid, asparagus fern, narcissus, tree bonsai and so on, the importance of this kind of decoration is not the $n$ umber, but their effect that 
make the finishing point. Therefore, decoration size and number should form a perfect ration with the living room space and furniture size to strengthen unity. In addition, the decoration volume should be not two big, and number should be small, try to coordinate the shapes. If meet mixed match decoration, they must be in order. The Neo-Chinese decorations must be coordinated with living room space environment.

\section{Design of lights in the living room}

Lights of the Neo-Chinese style must be oriented according to the while style, such as choose some archaize furniture, of course also can choose innovated, fashion and elegant one. There are many light types, ceiling lamp, droplight, wall lamp, floor lamp and craft candle; analyze from light texture, mainly include crystal, metal, plastic and wooden. Generally speaking, the main function of lights is lighting and decoration, another one is to foil atmosphere of the living room. When design light shape and texture, designers need to consider its space and main function. While no matter how to design, designers must pay attention to its integration with the whole decoration style. On the other hand, no matter decoration or main furniture, the function of these items is to make people feel comfortable. Therefore in the process of choosing Neo-Chinese style lights, designers must pay attention to the shape and functions of lights, must be coordinated with surrounding environment. Light source can choose LED lamp holder, this kind of lamp is not dazzling, saving energy and protecting environment, make people feel comfortable, warm and soft.

\section{Design of living room fabrics}

The design of fabrics in the soft assembly is also very important. The beautiful curves of fabrics can create a comfortable indoor atmosphere to make the indoor space not hard and empty. But creating a more tender, romantic, fresh and elegant, magnificent style, color of the fabrics should pay attention to the harmony with indoor color system, that is to say, the style of sofa, curtain, carpet should be integrated. Indoor decoration of the Neo-Chinese style can use natural plants pictures, auspicious pattern like prosperity brought by the dragon and the phoenix, cushion and sofa can use flaxen natural cloth or exquisite silk fabric, also can use wall paper with light landscape, this kind of decoration can make space looks fresh and elegant. If use fabrics with flowers, birds, fish(es) and insects pictures on sofa and window, can make people feel full of vigor. Wall design can use underslung decoration painting or hand embroidery with auspicious pattern.

For example, match a cushion for sofa, the cushion can be attractive.

\section{Furniture design}

Furniture design in the Neo-Chinese style mainly use post-modernism design, while traditional Chinese furniture has elegant quality, to recombine traditional furniture, to express through new nation culture to make people feel more flexible. Furniture design of Neo-Chinese style is very exquisite, lines are smooth, when determined Chinese style, designers need to avoid boring, tedious and preciseness. The popular pattern of modern Chinese style is traditional theme and modern material.

\section{Design principle of soft assembly decoration}

\section{The principle to strengthen culture theme and context of artistic conception}

No matter simple design or complicated design has their own context system, this kind of context expressed culture connotation. The Neo-Chinese decorations also have a certain emotion that around this culture theme. Only in this way can create typical culture space, not just help people associate in visual, but also can arouse peoples' emotional resonance. 


\section{Abide by aesthetic habits of different regions}

Different nations are with different religious faith, different territory, different education background, different characters, different interests and different taboos, soft assembly decoration must abide by proprietors' aesthetic habits.

\section{The whole function of spatial art should integrate with aesthetic design}

The Chinese style space pays attention to etiquette, therefore, designers must pay attention to sense of depth, such as beauty of symmetry, external isolation, hierarchy, respect for seniority and don on. On spatial art, we chase for visual beauty, on philosophy, it showed moderation. Though it is just a small decoration in the living room, it can be placed freely, but better pay attention to corresponding. For example, there is a Chinese window piece, thus there must have corresponding elements in the room to match with it, even in the washroom, such as blue and white porcelain washbasin. Corresponding and design makes the space be with sense of depth.

\section{Abide by the beauty sense of Chinese style that reservation and quiet}

The concept of Chinese style is to chase for beauty of connotation and the quiet of Zen. Solid space include wall, floor, soft decoration is a broaden of this kind of solid space, that is the so-called "beyond". Though this is no specific image, but through contrast of solid and void to open a "void" space to make an atmosphere.

\section{Well-proportioned}

The Neo-Chinese style space pays much attention on "human", people pay great attention to the order of layout, only this can conform people's living habit and mental habit. Generally speaking, when people look at spatial items, normally are in the circle from bottleneck to exciting to peace. From this side of the space to another side, soft assembly plays a very good guiding role. Such as use dynamic element (screen) to realize succession; cushion has a function of serving as a link between past and future. Therefore, soft decoration should be better in the position needs highlight. Such as to place a terracotta warrior in the end of corridor or the corner in the home, to make former bored environment become vivid and energetic to make space arrive a high tide and introduce to the next foreshadowing.

\section{References}

[1] Zhou Chengmin, Wang Qin. Discussion on the Market Design Principle of Home Soft Decoration - Set Modern Chinese Furniture Decoration Design as an Example, Furniture and Interior Decoration, 2011,10:67-69.

[2] Cheng Kun. Discussion on the Important Function of Soft Decoration in Interior Design, China Education of Light Industry, 2012,06:33-34+40.

[3] Lei Zhanshu. Discussion on the Value of Soft Decoration on Interior Design, Technology Outlook, 2014,06:9-11.

[4] Sun Shuang. Fan Siyi. Analysis on the Collocation Method of Soft Decoration on Interior Design, Modern Decoration (Theory), 2016,05:18.

[5] Chen Zicong, Zeng Tao. Application of the Neo-Chinese Style in Modern Interior Design,. Technology Wind, 2016,12:99-100.

[6] Zhou Chenmin, Wang Qin. Discussion on the Market Design Principle of Home Soft Decoration - Set Modern Chinese Furniture Decoration Design as an Example, Furniture and Interior Decoration, 2011,10:67-69. 